\title{
Single Nucleotide Polymorphism in Adiponectin Gene and Risk of Pancreatic Adenocarcinoma
}

\author{
Amal Ahmed Mohamed', Heba Omar², Mohamed F Abdel Ghaffar ${ }^{3}$, Mohamad \\ Saeed Marie ${ }^{2 *}$, Mohamed Ezz El Arab Ramadan ${ }^{4}$, Soha M Talima ${ }^{5}$, Mostufa EL \\ Daly $^{5}$, Seham Mahmoud ${ }^{6}$
}

\begin{abstract}
Introduction: Adiponectin is anti-inflammatory and anti-tumor cytokine secreted exclusively from adipocytes. There is a growing evidence of association between adiponectin gene polymorphism and development of pancreatic cancer. The current study aimed at evaluation of the possible association between selected adiponectin gene polymorphism and the risk of pancreatic cancer. Methods: Prospective case-control study included 77 patients (29 women and 48 men) with biopsy-proven pancreatic adenocarcinoma and 97 healthy control. Blood samples from all included participants were genotyped for 3 single nucleotide polymorphism (SNPs) of adiponectin genes ( $r s 1501299 \mathrm{C}>\mathrm{A}, \mathrm{rs} 266729 \mathrm{C}>\mathrm{G}$ and rs $2241766 \mathrm{G}>\mathrm{T}$ ) by PCR. Clinical, biochemical, and radiological data analyzed. Results: We demonstrated a significant association between the three studied SNPs (rs1501299, rs266729, and rs2241766) and increased risk of pancreatic adenocarcinoma $(\mathrm{p}<0.001)$. Furthermore, in clinical correlation analysis, Patients with rs 2241766 polymorphism have a lower frequency of lymph node involvement ( $p$ 0.05). Smoking and older age were independent predictors of pancreatic adenocarcinoma. Conclusion: We provided evidence that variants in adiponectin gene might influence the development and progression of pancreatic cancer.
\end{abstract}

Keywords: Pancreatic- adenocarcinoma- SNP- polymorphism- adiponectin

Asian Pac J Cancer Prev, 20 (1), 139-143

\section{Introduction}

Pancreatic adenocarcinoma is considered less common among other solid organ malignancies, ranked $11^{\text {th }}$ in both sexes (Ferlay et al., 2015), however, it has more aggressive and lethal course usually because of late-stage diagnosis (Jemal et al., 2005). Estimated 5-year survival rate ranges from 0 to $20 \%$ based on tumor stage at the time of diagnosis, respectability of the tumor and presence or absence of distant metastasis (Poruk et al., 2012).

Pancreatic cancer carries a diagnostic challenge; most of the diagnostic markers lack high sensitivity and specificity accuracy (Divella et al., 2017). Currently testing the susceptibility genes for pancreatic cancer is being studied to help early and more accurate diagnosis.

Adiponectin is the most important of a group of proteins collectively named as adipocytokines derived from adipose tissues in the body and its concentration in blood is inversely related to the body mass index (Ukkola and Santaniemi, 2002). It has a well-established role in regulating cell growth and inflammatory process also several disorders associated with insulin resistance (Yang et al., 2007). Recently its effect on tumor development is being studied in different types of cancer mainly breast and colon (An et al., 2012; Erbay et al., 2016), however, its role in cancer pancreas is still deficient.

Variations in adiponectin levels could be the result of genetic variations in the adiponectin (ADIPOQ) gene that is located on chromosome $3 \mathrm{q} 27$ thus affects its activity/expression. A number of SNPs in the ADIPOQ gene are associated with lower adiponectin expression or a predisposition to several diseases encoding adiponectin (Hivert et al., 2008).

The present study genotyped 3 SNPs at ADIPOQ genes namely rs $1501299 \mathrm{C}>\mathrm{A}, \mathrm{rs} 2241766 \mathrm{C}>\mathrm{G}$ and rs $266729 \mathrm{G}>\mathrm{T}$ to evaluate the possible association these SNPs and the risk of development of pancreatic adenocarcinoma in Egyptian cohort. 


\section{Materials and Methods}

\section{Patients and methods}

The present case-control study included 174 subjects: 77 with biopsy-proven pancreatic adenocarcinoma (48 men and 29 women; age, 44-76 years) and 97 gender and age matched healthy controls. This was a multicenter study, analyzed patients were hospitalized in Tanta University Faculty of Medicine and Ahmed Maher Teaching Hospital between 2012 and 2017. This study was carried out in accordance with the guidelines of the declaration of Helsinki and its subsequent amendments. The study protocol was approved by the ethical committee of Tanta University Faculty of Medicine. Written informed consent was obtained from each participant before study procedure.

Patients were suspected to have pancreatic adenocarcinoma based on clinical presentation with abdominal pain, jaundice, or weight loss or based on the presence of an incidental pancreatic mass on imaging. The diagnosis of pancreatic adenocarcinoma was confirmed by fine needle aspiration biopsy through percutaneous route (under US or CT guidance) or EUS-guided. Tumor resectability were defined on a continuum from resectable to unresectable according to the involvement of adjacent structures and the presence of distant metastases.

All participants completed the full history taking, clinical examination, and laboratory investigations (AST, ALT, Alkaline phosphatase, GGT, albumin, bilirubin, CA19-9) using conventional automated analyzers in the Biochemistry Laboratory at National Hepatology \&Tropical Medicine Research -. All biochemical analyses were performed in a "blinded" manner.

\section{DNA Extraction}

Genomic DNAwas extracted fromEDTA-anticoagulated peripheral blood leucocytes using QIA amp DNA Blood Mini Kit supplied by Qiagen GmbH (Hiden, Germany) (Schur et al., 2001). The DNA concentration was determined from the absorbance at $260 \mathrm{~nm}$. All samples had a 260/280 nm absorbance ratio between 1.59 and 1.77 indicating the purity of DNA. The integrity of the DNA was checked by electrophoresis on $0.8 \%$ agarose gel stained with ethidium bromide.

\section{Gene polymorphism}

We chose to study ADIPOQ gene SNPs; rs1501299, rs266729, and rs2241766 based on evidence from the previous studies that found a possible association between previously mentioned SNPs and cancer due to their impact on serum adiponectin (divella et al., 2017; Kaklamani et al., 2008).

\section{Detection of gene polymorphism}

DNA from patients was extracted from venous blood using standard methods. Genotyping of the ADIPOQ gene SNPs; rs1501299, rs266729, and rs2241766 based on evidence from the previous rs was performed using a TaqMan 5' allelic discrimination assay (Applied Biosystems). The probes were labeled with the fluorescent dyes VIC and FAM respectively.
As the Taq polymerase extends the primer and synthesizes the nascent strand, the 5' to 3' exonuclease activity of the Taq polymerase degrades the probe that has annealed to the template. Degradation of the probe releases the fluorophore from it and breaks the close proximity to the quencher, thus relieving the quenching effect and allowing fluorescence of the fluorophore. Hence, fluorescence detected in the quantitative PCR thermal cycler is directly proportional to the fluorophore released and the amount of DNA template present in the PCR. Genotyping of each sample was automatically attributed for allelic discrimination.

\section{Statistical analyses}

Data were entered, validated, and analyzed using STATA 14 software. Patients' demographic and routine laboratory values data were expressed as number (percent) for categorical variables and as mean $( \pm S D)$ for continuous variables. Comparisons between pancreatic adenocarcinoma cases and controls were done using students' t-test or Mann-Whitney test whenever appropriate. Chi-square test was used for comparison of categorical data. Associations between clinical characteristics, polymorphism, and adenocarcinoma diagnosis were investigated using chi-square test. Univariate and multivariate logistic regression models were constructed to identify baseline predictors of pancreatic adenocarcinoma. Data were reported as odds ratios (OR) with 95\% Confidence Intervals (95\% CI). All statistical analyses were based on two-sided hypothesis tests with a significance level of $\mathrm{p}<0.05$.

\section{Results}

Seventy-seven patients with biopsy-proven pancreatic adenocarcinoma, as well as, 97 normal age and gender-matched control were evaluated in the current study. Patients with pancreatic adenocarcinoma were significantly older than controls $(62.47 \pm 6.89$ vs. 55 $\pm 12.68, \mathrm{p}<0.001)$. There were significantly more ever smokers and current smokers among cases compared to the controls (42.86 vs. $19.59 \%$; $<<0.01$ ). Positive family history of pancreatic tumors was significantly higher in patients with pancreatic tumor in comparisons to controls $(22.08 \%$ vs. $3.09 \%, \mathrm{p}<0.001)$. A total of 42 patients $(54.55 \%)$ presented with abdominal pain. Baseline laboratory data of included participants are depicted in Table .1.

In patients with pancreatic adenocarcinoma, the tumor size ranged from 1.8 to $5.5 \mathrm{~cm}$ (mean, $3.22 \pm 1.0$ ). Local vascular invasion was found in 22 patients $(28.57 \%)$. Lymph nodes and liver metastases were observed in 25 $(32.47 \%)$ and $36(46.75 \%)$ patients respectively (Table 2 ).

The genotype distributions of SNPs of adiponectin gene (ADIPOQ) between patients with pancreatic cancer and control are summarised in Table 3 . There was a significant association between the three studied SNPs (rs1501299, rs266729 and rs2241766) and development of pancreatic adenocarcinoma $(\mathrm{p}<0.001)$.

The potential association between the SNPs of ADIPOQ gene (rs1501299, rs266729, and rs2241766) and 
DOI:10.31557/APJCP.2019.20.1.139

SNPs in Adiponectin Gene and Risk of Pancreatic Adenocarcinoma

Table 1. Baseline Demographic and Clinical and Laboratory Data, n=174

\begin{tabular}{|c|c|c|c|}
\hline & Control $\mathrm{n}=97$ & Pancreatic cancer $n=77$ & $P$ value \\
\hline \multicolumn{4}{|l|}{ Age } \\
\hline Mean \pm SD & $55 \pm 12.68$ & $62.47 \pm 6.89$ & $<0.001$ \\
\hline Range & $37-80$ & $44-76$ & \\
\hline Male gender & $51(52.58)$ & $48(62.34)$ & 0.2 \\
\hline BMI $\left(\mathrm{kg} / \mathrm{m}^{2}\right)$ & $30.41 \pm 9.25$ & $30.19 \pm 11.29$ & 0.88 \\
\hline Rural, n (\%) & $25(25.77)$ & $52(67.53)$ & \\
\hline Urban, n (\%) & $72(74.23)$ & $25(32.47)$ & $<0.001$ \\
\hline Smoking (yes), n (\%) & $19(19.59)$ & $33(42.86)$ & 0.001 \\
\hline Abdominal pain (yes), n (\%) & $2(2.06)$ & $42(54.55)$ & $<0.001$ \\
\hline Fever, (yes), n (\%) & $2(2.06)$ & $17(22.08)$ & $<0.001$ \\
\hline Positive family history of pancreatic cancer (yes), n (\%) & $3(3.09)$ & $17(22.08)$ & $<0.001$ \\
\hline AST & $42.12 \pm 37.73$ & $150.40 \pm 58.85$ & $<0.001$ \\
\hline ALT & $46.53 \pm 30.83$ & $72.65 \pm 53.53$ & $<0.001$ \\
\hline Median ALP(IQR) & $77(57-96)$ & $4550(356-560)$ & $<0.001$ \\
\hline Median GGT (IQR) & $40(30-55)$ & $260(200-401)$ & $<0.001$ \\
\hline Total bilirubin & $0.82 \pm 0.26$ & $8.35 \pm 6.26$ & $<0.001$ \\
\hline Albumin & $3.65 \pm 0.50$ & $3.42 \pm 0.68$ & 0.02 \\
\hline INR & $1.01 \pm 0.11$ & $1.36 \pm 0.18$ & $<0.001$ \\
\hline Blood sugar & $111.49 \pm 39.37$ & $213.30 \pm 144.59$ & $<0.001$ \\
\hline Triglycerides & $173.45 \pm 59.35$ & $203.14 \pm 76.53$ & 0.005 \\
\hline Cholesterol & $176.33 \pm 72.96$ & $248.39 \pm 163.88$ & 0.0002 \\
\hline Median AFP(IQR) & $6.8(5-17)$ & $230(20-452)$ & $<0.001$ \\
\hline Ca19-9 & $28.77 \pm 30.66$ & $270.57 \pm 260.09$ & $<0.001$ \\
\hline
\end{tabular}

Table 2. Characteristics of Pancreatic Tumor, $n=77$

\begin{tabular}{lc}
\hline Variable & Value \\
\hline Tumor size & $3.22 \pm 1.0$ \\
Lymph node metastasis yes, n (\%) & $25(32.47)$ \\
Vascular invasion (yes), n (\%) & $22(28.57)$ \\
Distant metastasis (yes), n (\%) & $36(46.75)$ \\
Resectability (yes), n (\%) & $20(25.97)$ \\
\hline
\end{tabular}

the clinical data of pancreatic adenocarcinoma patients were investigated. Male gender, as well as older age, was significantly associated with rs2241766 polymorphism (p 0.02 and 0.06 respectively). Patients with rs 2241766 polymorphism have a lower frequency of lymph node involvement ( $\mathrm{p}$ 0.05) as shown in table. 4

Univariate and multivariate-adjusted logistic regression models were constructed to identify baseline predictors of pancreatic adenocarcinoma. Older age (p-value 0.03), smoking (p-value 0.04) and polymorphism of ADIPOQ gene (rs1501299, rs266729 and rs2241766) (p-value $<0.0001$ ) were independent predictors of pancreatic adenocarcinoma (Table 5).

\section{Discussion}

Pancreatic adenocarcinoma incidence has been linked to several factors, of which serum adiponectin level was recently evaluated with varying results (Rückert et al., 2010). In our study, we evaluated 3 SNPs in adiponectin gene (at rs1501299, rs266729, and rs2241766) and its relation to the development of pancreatic adenocarcinoma.

We demonstrated a higher frequency of rs 1501299A $>C$ in patients with pancreatic cancer (compared to control group (OR 3.14 95\% CI 1.68-5.87). This was different from a protective effect of such change that was observed in Chinese population-based study by (Yang et al., 2015) also from Japanese population in a study by (Kuruma et al., 2014) who showed 1.9 folds increased pancreatic

Table 3. Distribution of SNPs between Patients with Pancreatic Cancer and Control

\begin{tabular}{llcccc}
\hline & & Control $\mathrm{n}=97$ & Pancreatic cancer $\mathrm{n}=77$ & OR $(95 \%$ CI $)$ & P value \\
\hline rs1501299 & CC & $67(69.07)$ & $32(41.56)$ & $0.48(0.31-0.73)$ & $<0.001$ \\
& CA or AA & $30(30.93)$ & $45(58.44)$ & $3.14(1.68-5.87)$ & $<0.001$ \\
rs266729 & CC & $67(69.07)$ & $31(40.26)$ & $0.46(0.30-0.71)$ & $<0.001$ \\
& CG or GG & $30(30.93)$ & $46(59.74)$ & $3.31(1.77-6.20)$ & $<0.001$ \\
rs2241766 & GG & $72(74.23)$ & $37(48.05)$ & $0.51(0.35-0.76)$ & $<0.001$ \\
& GT or TT & $25(25.77)$ & $40(51.95)$ & $3.11(1.65-5.89)$ & $<0.001$ \\
\hline
\end{tabular}


Table 4. Association between SNPs of ADIPOQ Gene and Clinical Features of the Tumor in Pancreatic Cancer Cases, $\underline{\mathrm{n}=77}$

\begin{tabular}{|c|c|c|c|c|c|c|c|}
\hline & \multicolumn{2}{|c|}{ rs1501299 } & \multicolumn{2}{|c|}{ rs266729 } & \multicolumn{2}{|c|}{ rs2241766 } & \multirow[t]{2}{*}{$\mathrm{P}$} \\
\hline & $\mathrm{CC}$ & $\mathrm{CA}$ or $\mathrm{AA}$ & $\mathrm{CC}$ & $\mathrm{CG}$ or $\mathrm{GG}$ & GG & GT or TT & \\
\hline Age, years & & & & & & & $* 0.473$ \\
\hline$<60$ & $13(37.14)$ & $22(62.86)$ & $12(34.29)$ & $23(65.71)$ & $22(62.86)$ & $13(37.14)$ & $* * 0.329$ \\
\hline$>60$ & $19(45.24)$ & $23(54.76)$ & $19(45.24)$ & $23(54.76)$ & $15(35.71)$ & $27(64.29)$ & $\# 0.02$ \\
\hline Gender & & & & & & & $* 0.33$ \\
\hline Female & $10(34.48)$ & $19(65.52)$ & $10(34.48)$ & $19(65.52)$ & $18(62.07)$ & $11(37.93)$ & $* * 0.42$ \\
\hline Male & $22(45.83)$ & $26(54.17)$ & $21(43.75)$ & $27(56.25)$ & $19(39.58)$ & $29(60.42)$ & $\# 0.06$ \\
\hline BMI & & & & & & & $* 0.74$ \\
\hline$<25$ & $18(40)$ & $27(60)$ & $15(33.33)$ & $30(66.67)$ & $21(46.67)$ & $24(53.33)$ & $* * 0.14$ \\
\hline$>25$ & $14(43.75)$ & $18(56.25)$ & $16(50)$ & $16(50)$ & $16(50)$ & $16(50)$ & $\# 0.77$ \\
\hline Smoking & & & & & & & $* 0.74$ \\
\hline Yes & $19(43.18)$ & $25(56.82)$ & $18(40.91)$ & $26(59.09)$ & $22(50)$ & $22(50)$ & $* * 0.89$ \\
\hline No & $13(39.39)$ & $20(60.61)$ & $13(39.39)$ & $20(60.61)$ & $15(45.45)$ & $18(54.55)$ & $\# 0.69$ \\
\hline Tumor size & & & & & & & $* 0.55$ \\
\hline$<3 \mathrm{~cm}$ & $22(44)$ & $28(56)$ & $20(40)$ & $30(60)$ & $22(44)$ & $28(56)$ & $* * 0.995$ \\
\hline $3 \mathrm{~cm}$ & $10(37.04)$ & $17(62.96)$ & $11(40.74)$ & $16(59.26)$ & $15(55.56)$ & $12(44.44)$ & $\# 0.33$ \\
\hline Vascular invasion & & & & & & & $* 0.56$ \\
\hline no & $24(43.64)$ & $31(56.36)$ & $21(38.18)$ & $34(61.82)$ & $24(43.64)$ & $31(56.36)$ & $* * 0.56$ \\
\hline yes & $8(36.36)$ & $14(63.64)$ & $10(45.45)$ & $12(54.55)$ & $13(59.09)$ & $9(40.91)$ & $\# 0.22$ \\
\hline Lymph nodes meta & tasis & & & & & & $* 0.24$ \\
\hline no & $24(46.15)$ & $28(53.85)$ & $19(36.54)$ & $33(63.46)$ & $21(40.38)$ & $31(59.62)$ & $* * 0.34$ \\
\hline yes & $8(32)$ & $17(68)$ & $12(48)$ & $13(52)$ & $16(64.00)$ & $9(36)$ & $\# 0.05$ \\
\hline Distant metastasis & & & & & & & $* 0.35$ \\
\hline no & $15(36.59)$ & $26(63.41)$ & $20(48.78)$ & $21(51.22)$ & $21(51.22)$ & $20(48.78)$ & $* * 0.10$ \\
\hline yes & $17(47.22)$ & $19(52.78)$ & $11(30.56)$ & $25(69.44)$ & $16(44.44)$ & $20(55.56)$ & $\# 0.55$ \\
\hline Resectability & & & & & & & $* 0.87$ \\
\hline Non & $24(42.11)$ & $33(57.89)$ & $22(38.60)$ & $35(61.40)$ & $25(43.86)$ & $32(56.14)$ & $* * 0.62$ \\
\hline Resectable & $8(40.00)$ & $12(60.00)$ & $9(45.00)$ & $11(55.00)$ & $12(60)$ & $8(40)$ & $\# 0.21$ \\
\hline CA19-9 & & & & & & & *0.19 \\
\hline$<37$ & $4(66.67)$ & $2(33.33)$ & $2(33.33)$ & $4(66.67)$ & $3(50)$ & $3(50)$ & $* * 0.72$ \\
\hline$>37$ & $28(39.44)$ & $43(60.56)$ & $29(40.26)$ & $42(59.15)$ & $34(47.89)$ & $37(51.95)$ & $\# 0.92$ \\
\hline
\end{tabular}

*, p value for patients with rs1501299 CC vs. CA or AA; **, p value for patients with rs266729 CC vs. CG or GG; \#, p value for patients with rs2241766 GG vs. GT or TT.

Table 5. Univariate and Multivariate Adjusted Logistic Regression Model forBaseline Predictors of Pancreatic Carcinoma

\begin{tabular}{lcccc}
\hline Variable & Univariate & \multicolumn{2}{c}{ Multivariate } \\
& OR $(95 \% \mathrm{CI})$ & P value & OR (95\% CI) & P value \\
\hline Age $>60$ & $3.11(1.66-5.85)$ & $<0.001$ & $2.34(1.10-4.99)$ & 0.03 \\
Male gender & $1.49(0.81-2.75)$ & 0.2 & $1.21(0.57-2.58)$ & 0.62 \\
Smoking & $3.08(1.57-6.05)$ & $<0.001$ & $2.28(1.04-4.96)$ & 0.04 \\
BMI $>25$ & $0.85(0.47-1.57)$ & 0.62 & $1(0.48-2.06)$ & 1 \\
rs1501299 $(\mathrm{CA}$ or AA) & $3.14(1.68-5.87)$ & $<0.001$ & $2.92(1.42-6.00)$ & $<0.001$ \\
rs266729 $(\mathrm{CG}$ or GG) & $3.31(1.77-6.20)$ & $<0.001$ & $3.68(1.79-7.56)$ & $<0.001$ \\
rs2241766 $(\mathrm{GT}$ or TT) & $3.11(1.65-5.89)$ & $<0.001$ & $2.63(1.27-5.44)$ & 0.01 \\
\hline
\end{tabular}

cancer risk in patients with CC genotype compared to AA genotype. This in part could be explained by the different ethnic groups.

Furthermore, several studies plotted significant correlation of SNP rs1501299 with characters of the tumor; importantly the tumor size (Yang et al., 2015). In our study apart from a significantly lower frequency of lymph node involvement in patients with rs2241766 
polymorphism, we didn't find significant correlation to the tumor size.

To our knowledge SNPs at rs2241766 and rs266729 were studied in different metabolic and neoplastic disorders including T2DM, colon and prostate cancer through their role on serum adiponectin level (Yang et al., 2015; Kaklamani et al., 2011). Their role is not widely studied in patients with pancreatic adenocarcinoma. rs2241766 $\mathrm{G}>\mathrm{T}$ change and $\mathrm{rs} 266729 \mathrm{C}>\mathrm{G}$ change were more represented in patients with pancreatic adenocarcinoma compared to control; OR (95\% CI) $3.11(1.65-5.89)$ and $3.31(1.77-6.20)$ respectively, thus carrying an increased potential risk for pancreatic cancer.

In addition to studied SNPs and according to univariate and multivariate-adjusted logistic regression models, older age and smoking were independent predictors of pancreatic adenocarcinoma which match results by (Bosetti et al., 2011) that showed ongoing cigarette smoking carries a twofold increased risk of pancreatic cancer and that the risk increases with higher smoking index.

We didn't test serum adiponectin level in our patients; this can be considered as a drawback and limitation of our study. Further studies to assess adiponectin level in patients with pancreatic adenocarcinoma and its relation to different adiponectin genotypes can help to assess underlying pathogenesis. Also, our research was restricted in the genetic variants analysis to a relatively small scale Egyptian population, so larger-scale of patients and distinct population samples should be taken for more results validation.

In conclusion, SNPs at adiponectin genes (rs1501299, rs266729 and rs2241766) are associated with increased risk of cancer pancreas. Multi-ethnic large-scale studies are needed to confirm our results.

\section{Disclosure of Funding Source \\ None.}

\section{Ethical approval}

This study was approved by ethical committee of Tanta University Faculty of Medicine, Egypt.

\section{Conflict of interest}

None.

\section{References}

An W, Bai Y, Deng SX, et al (2012). Adiponectin levels in patients with colorectal cancer and adenoma: a meta-analysis. Eur $J$ Cancer Prev, 21, 126-33.

Bhat K, Wang F, Ma Q, et al (2012). Advances in biomarker research for pancreatic cancer. Curr Pharm Des, 18, 2439-51.

Bosetti C, Lucenteforte E, Silverman T, et al (2012). Cigarette smoking and pancreatic cancer: an analysis from the International Pancreatic Cancer Case-Control Consortium (Panc4). Ann Oncol, 23, 1880-8.

Divella R, Daniele A, Mazzocca A, et al (2017). ADIPOQ rs266729 G/C gene polymorphism and plasmatic adipocytokines connect metabolic syndrome to colorectal cancer. J Cancer, 8, 1000-8.
Erbay B, Y1lmaz TU, Eraldemir C, et al (2016). The relationship between adiponectin and breast cancer. J Breast Health, $12,67-71$.

Ferlay J, Soerjomataram I, Ervik M, et al (2015). Cancer incidence and mortality worldwide: sources, methods and major patterns in GLOBOCAN 2012. Int J Cancer, 136, 359-86

Hivert MF, Manning AK, McAteer JB, et al (2008). Common variants in the adiponectin gene (ADIPOQ) associated with plasma adiponectin levels, type 2 diabetes, and diabetes-related quantitative traits: the Framingham Offspring Study. Diabetes, 57, 3353-9

Jemal A, Murray T, Ward E, et al (2005). Cancer statistics. $C A$ Cancer J Clin, 55, 10-30.

Kaklamani V, Sadim M, Hsi A, et al (2008). Variants of the adiponectin and adiponectin receptor 1 genes and breast cancer risk. Cancer Res, 68, 3178-84.

Kaklamani V, Yi N, Zhang K, et al (2011). Polymorphisms of ADIPOQ and ADIPOR1 and prostate cancer risk Metabolism, 60, 1234-43.

Kuruma S, Egawa N, Kurata M, et al (2014). Case-control study of diabetes-related genetic variants and pancreatic cancer risk in Japan. World J Gastroenterol, 20, 17456-62.

Poruk KE, Firpo MA, Adler DG, Mulvihill SJ (2012). Screening for pancreatic cancer: why, how, and who? Ann Surg, 257, 17-26.

Rückert F, Christian Pilarsky C, Grützmann R (2010). Serum tumor markers in pancreatic cancer. Cancer Discov, 2, 1107-24

Schur BC, Bjereke J, Nuwayhid N, Wong SH (2001). Genotyping of cytochrome P450 2D6*3 and *4 mutations using conventional PCR. Clin Chem Acta, 308, 25-31.

Ukkola O, Santaniemi M (2002). Adiponectin: a link between excess adiposity and associated comorbidities?. J Mol Med, 80, 696-702.

Yang WS, Yang YC, Chen CL, et al (2007). Adiponectin SNP276 is associated with obesity, the metabolic syndrome, and diabetes in the elderly. Am J Clin Nutr, 86, 509-13.

Yang J, Li X, Wang F, et al (2015). Association analysis of genetic variants of adiponectin gene and risk of pancreatic cancer. Int J Clin Exp Med, 8, 8094-8100

Yang X, Li J, Cai W, et al (2015). Adiponectin gene polymorphisms are associated with increased risk of colorectal cancer. Med Sci Monit, 21, 2595-2606.

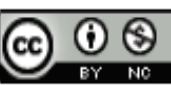

This work is licensed under a Creative Commons AttributionNon Commercial 4.0 International License. 